\title{
Influence of the left-handed part of the neutrino mass matrix on the lepton number violating $e^{-} e^{-} \rightarrow W^{-} W^{-}$process
}

\author{
P. Duka, J. Gluza, M. Zrałek \\ Department of Field Theory and Particle Physics Institute of Physics, University of Silesia Uniwersytecka 4, PL-40-007 \\ Katowice, Poland E-mails: duka,gluza,zralek@us.edu.pl
}

(August 11, 2018)

\begin{abstract}
Influence of the neutrino mass submatrix $M_{L}$ on the $e^{-} e^{-} \rightarrow W^{-} W^{-}$process is discussed. Taking into account various possible CP signatures of heavy neutrinos it is shown that, in some cases, nonzero $M_{L}$ substantially changes predictions for maximum possible values of the $e^{-} e^{-} \rightarrow W^{-} W^{-}$ cross section. A direct role of the $\omega^{2}$ parameter (coming from neutrinoless double beta decay) is clarified. The consequences of doubly charged Higgs particles $\left(\delta^{--}\right)$with resonances even far away from energies of the future linear lepton collider $(\sqrt{s}=0.5 \div 1 \mathrm{TeV})$ are studied.
\end{abstract}

13.15.-f,12.15.Cc,11.30.Er

\section{INTRODUCTION}

The $e^{-} e^{-}$option of the future $\sqrt{s}=0.5 \div 2 \mathrm{TeV}$ linear collider is a very interesting area for investigating new physics [1]. Processes like $e^{-} e^{-} \rightarrow l_{i}^{-} l_{j}^{-}, W^{-} W^{-}\left(l_{i(j)}=e, \mu, \tau\right)$ could be studied indicating that lepton number (flavour or total) is not a global symmetry of the electroweak interactions. In this paper we would like to examine the $e^{-} e^{-} \rightarrow W^{-} W^{-}$process. This reaction violates the total lepton number by two units, $\Delta L=2$. Its potential importance and hopes connected with it are based on two facts. First, the SM background is very small and under control [2] so with the planed luminosity $10 \mathrm{fb}^{-1} /$ year [1] the cross section as small as $0.1 \mathrm{fb}$ could give visible effect. Second, its occurrence would indicate that there exist massive neutrinos of Majorana type. These neutrinos must be heavy (with masses $M_{N}>M_{Z}$ ) as known neutrinos cannot give any substantial signal [3]. Many papers have been devoted to this process during the last decade [4 [7]. For the first time this reaction was proposed and examined in 1982 by Rizzo [ [ 1 . Additional interest has come with the paper [5] where it was shown that the process is enhanced for heavy neutrino masses in the vicinity of the collider's c.m. energy. Then the optimism was revised in [6] where constraints on heavy neutrinos coming from neutrinoless double- $\beta$ decay have been taken into account. It has been shown that an observable signal requires fine-tuning among different heavy neutrino couplings. However, as it has been shown in [8], these cancelations can be in a natural manner connected with CP parities of heavy neutrinos. All other papers cited in [7] give many interesting details connected with the process.

This paper brings another such a detail which can however appear to be crucial for the magnitude of the cross section. In the last paper concerning the $e^{-} e^{-} \rightarrow W^{-} W^{-}$where all relevant constraints on the heavy neutrinos have been taken into account [8] we have assumed that the neutrino mass submatrix $M_{L}$ generated by left-handed neutrinos

$$
M=\left(\begin{array}{ll}
M_{L} & M_{D} \\
M_{D}^{T} & M_{R}
\end{array}\right),
$$

is exactly zero $\left(M_{D}\right.$ is the submatrix of Dirac type masses, $M_{R}$ is the submatrix of right-handed Majorana masses). This means that we have considered the class of models beyond the standard where only right-handed neutrinos were introduced. Then, to get an observable magnitude of the cross section, at least 3 heavy neutrinos with appropriate $\mathrm{CP}$ signatures and masses were necessary. However, there are models where $M_{L}$ does not vanish. Such nonzero $M_{L}$ changes the relations which restrict the space of parameters of possible (i.e. allowed by experimental data) heavy neutrinos couplings and masses.

The full phenomenological discussion of non-zero $M_{L}$ has been given lately [9] in the context of heavy neutrino production in $e^{-} e^{+}\left(e^{-} e^{+} \rightarrow \nu N\right)$ and $e^{-} \gamma\left(e^{-} \gamma \rightarrow W^{-} N\right)$ reactions. Here we will restrict ourselves to two nonstandard models with possible nonzero $M_{L}$. The Standard Model with both additional right handed neutrinos (RHS) and Higgs triplets and the Left-Right symmetric model (LR). Details of these models can be found in literature (e.g. in [3, 10]). As we are going to find the largest possible values of the cross section we consider models where CP is conserved in the lepton sector. 


\section{THE INFLUENCE OF $M_{L}$ ON $E^{-} E^{-} \rightarrow W^{-} W^{-}$.}

The leading helicity amplitudes for the $e^{-} e^{-} \rightarrow W^{-} W^{-}$process can be written in the following, simplified, way

$$
M=\sum_{a}\left\{K_{a e}^{2} m_{a}\left[f_{t}\left(m_{a}\right)+f_{u}\left(m_{a}\right)+f_{s}^{L}\right]+\left(K_{R}\right)_{a e}^{2} m_{a} f_{s}^{R}\right\} .
$$

The matrices $K, K_{R}$ are part of the unitary matrix $U=\left(K^{*}, K_{R}\right)^{T}$ which diagonalizes the $6 \times 6$ neutrino mass matrix $\mathrm{M}$ in Eq.(1), the index $\mathrm{L}(\mathrm{R})$ is connected with the left (right) doubly charged Higgs particle which is exchanged in the s channel. For details see e.g. [3], [10].

The sum in Eq. (2) runs over all light $(\nu)$ and heavy $(\mathrm{N})$ neutrinos. Let's note that the kinematical factors in $\mathrm{t}$ and $\mathrm{u}$ channels $f_{t, u}$ depend on $m_{a}$, but the $f_{s}^{L(R)}$ ones in s channel do not. First we will examine the $\mathrm{t}$ and $\mathrm{u}$ channels assuming only that the influence of the s channel is negligible (heavy $\delta_{L, R}^{-}$). At the end we will comment on the effect of non-zero $M_{L}$ on the s-channel contribution.

The experimental bounds on the elements of the matrix, $K_{\nu e}$ and $K_{N e}$, describing the mixing of electrons with light and heavy neutrinos can be summarized as follows

$$
\begin{gathered}
\sum_{N(\text { heavy })}\left|K_{N e}^{2}\right| \leq \kappa^{2}=0.0054 \\
\sum_{\nu(\text { light })}\left|K_{\nu e}^{2} m_{\nu}\right| \leq \kappa_{\text {light }}^{2}=0.65 \mathrm{eV} \\
\left|\sum_{N(\text { heavy })} K_{N e}^{2} \frac{1}{m_{N}}\right| \leq \omega^{2}=6 \cdot 10^{-3} \div 5 \cdot 10^{-5} \mathrm{TeV}^{-1} .
\end{gathered}
$$

The first relation (Eq.(3)) comes from low energy experiments [1], the other ones can be derived from the fact that neutrinoless double- $\beta$ decay $(\beta \beta)_{0 \nu}$ has not been detected yet ${ }^{1}$.

Diagonalization of the matrix (1) together with (4) yields to the following relation $\left(m_{L}=\left(M_{L}\right)_{\nu_{e} \nu_{e}}\right)$

$$
\left|m_{L}-\sum_{N} K_{N e}^{2} m_{N}\right|<\kappa_{\text {light }}^{2}
$$

However, $\kappa_{\text {light }}^{2}$ is very small and can be neglected, then from (6) we get

$$
\sum_{N} m_{N} K_{N e}^{2}=m_{L}
$$

Similar to the analysis given in [8],9] let us discuss the influence of $m_{L}$ on the magnitude of the cross section for different CP parities of heavy neutrinos.

If we have only one heavy neutrino state (or more but with the same CP parities) then from (3),(5) and (7) we get restrictions on $m_{L}$ [9]

$$
0 \leq m_{L} \leq \min \left(\kappa^{2} M, \omega^{2} M^{2}\right)
$$

where $\mathrm{M}$ is the mass of the lightest of heavy neutrinos. It gives for instance $m_{L} \leq 5 \cdot 10^{-4} \mathrm{GeV}$ for $\mathrm{M}=100 \mathrm{GeV}$ $\left(\omega^{2}=5 \cdot 10^{-5} \mathrm{TeV}^{-1}\right)$. For the above values of $m_{L}$ the mixing angle $K_{N e}$ is limited to [9]

$$
K_{N e}^{2} \leq \min \left(\omega^{2} M, \kappa^{2}\right)
$$

\footnotetext{
${ }^{1}$ As we can see there exist large discrepancies in the limit on $\omega^{2}$. For arguments on lower (upper) limits, see 12] (13]).
} 
Fig.1 shows the maximum value of the cross section $\sigma\left(e^{-} e^{-} \rightarrow W^{-} W^{-}\right)$where the parameters are restricted by relation (9) for three different values of $\omega^{2}$ and $\sqrt{s}=0.5(1) \mathrm{TeV}$. We can see that for various $\omega^{2}$ there are different masses $M_{0}$ for which the cross section reaches maximum value, e.g. $M_{0} \simeq 1(100) \mathrm{TeV}$ for $\omega^{2}=6 \cdot 10^{-3}\left(5 \cdot 10^{-5}\right) \mathrm{TeV}-1$. For $M \leq M_{0}$ the maximum value of the cross section increases with increasing $\mathrm{M}\left(\left(K_{N e}\right)_{\max }^{2}\right.$ in Eq.(9) increases), for masses larger than $M_{0}$ the cross section decreases with $\mathrm{M}\left(\left(K_{N e}\right)_{\max }^{2}=\kappa^{2}=\right.$ const $)$. We can see that only for $\omega^{2}>5 \cdot 10^{-4} \mathrm{TeV}^{-1}$ and $\sqrt{s} \geq 1 \mathrm{TeV}$ there is a small region of masses where $\sigma_{\max }>0.1 \mathrm{fb}$. If there is only one heavy neutrino or more but with the same CP parities then the value of $\omega^{2}$ crucially determines $\sigma_{\max }$. Much effort is devoted to find the bound on $\omega^{2}$ parameters [12].

For the case of two heavy neutrinos with opposite CP parities we get the following inequalities $\left(K_{N_{1} e}=x_{1}, K_{N_{2} e}=\right.$ $\left.i x_{2}, m_{1}=M, m_{2}=A M\right)$

$$
\begin{aligned}
x_{1}^{2}+\left|\frac{x_{1}^{2}}{A}-\frac{m_{L}}{A M}\right| & \leq \kappa^{2}, \\
\left|x_{1}^{2}\left(1-\frac{1}{A^{2}}\right)+\frac{m_{L}}{A^{2} M}\right| & \leq \omega^{2} M .
\end{aligned}
$$

When $m_{L}=0$, to remove the bound given by $\omega^{2}$ (Eq.(11)), we have to assume that two neutrinos are almost degenerate: $A \rightarrow 1$. But then we have practically one Dirac neutrino (two Majorana neutrinos with opposite CP values) and the cross section approaches zero. This was actually shown in [8] where the $m_{L}=0$ case was examined.

However, for $m_{L} \neq 0$ situation is different. The inequalities (10) and (11) can be satisfied only for the confined region of $m_{L}$ [9]

$$
-\max \left(A M \kappa^{2},(A-1) M \kappa^{2}+A^{2} \omega^{2} M^{2}\right) \leq m_{L} \leq \min \left(\omega^{2} M^{2}, \kappa^{2} M\right) .
$$

Positive values of $m_{L}$ are strongly restricted but the space of negative $m_{L}$ values is wider and depends on the values of $\mathrm{M}$ and A. In Fig.2 we plot the results for $m_{L}=-1(-3,-5) \mathrm{GeV}$ and $\mathrm{A}=5$ as a function of neutrino mass. As we can see lines start from different masses. This is because Eq.(12) must holds. Similar results can be obtained for larger spectrum of $\mathrm{A}(=3 \div 15)$. For positive $m_{L}$ the situation is similar to the case with $n_{R}=1$ (compare Eqs.(8),(12)). Results given in Fig.2 describe also the case of three heavy neutrinos with the following CP signatures $\eta_{C P}\left(N_{1}\right)=-\eta_{C P}\left(N_{2}\right)=-\eta_{C P}\left(N_{3}\right)$. Then two heavy neutrinos $\left(N_{2}, N_{3}\right)$ contribute in the same way to the amplitude (Eq.(2)) and can be effectively treated as one.

The last quantitatively distinguishable possibility which is left for three heavy neutrinos is the case $\eta_{C P}\left(N_{1}\right)=$ $\eta_{C P}\left(N_{2}\right)=-\eta_{C P}\left(N_{3}\right)$. Then initial inequalities $(3,5)$ and $(7)$ are satisfied if $m_{L}$ is confined to the following region $\left(m_{1}=M, m_{2}=A M, m_{3}=B M\right)[9]$

$$
\begin{array}{r}
-\min \left\{B M \kappa^{2}, \max \left\{B^{2} \omega^{2} M^{2},(B-1) M \kappa^{2}+B \omega^{2} M^{2}\right\}\right\} \leq m_{L} \\
\leq \min \left\{A M \kappa^{2},(A-B) M \kappa^{2}+A B \omega^{2} M^{2}\right\} .
\end{array}
$$

By fixing $\mathrm{B}=10$ and $\mathrm{M}=100 \mathrm{GeV}$ for different values of $\mathrm{A}$ we have found mixing angles $K_{N_{1} e}=x_{1}, K_{N_{2} e}=$ $x_{2}, K_{N_{3} e}=i x_{3}$ such that the cross section is maximal. The result is given in Fig.3 for $\sqrt{s}=1 \mathrm{TeV}$. For larger masses $(M>100 \mathrm{GeV}) \sigma_{\max }$ decreases, e.g. for $\mathrm{M}=200 \mathrm{GeV}, \sigma_{\max } \leq 4 \mathrm{fb}$. Let us note that the largest results are possible for large $\mathrm{A}$ and then we can always find a space of allowed mixings for which $\sigma_{\max } \simeq 9 \mathrm{fb}$ independently of $m_{L}$. Similar plots can be made for other energies $0.5 \mathrm{TeV} \leq \sqrt{s} \leq 2 \mathrm{TeV}$ with a result $\sigma_{\max } \leq 1(25,40) \mathrm{fb}$ and $\sqrt{s}=0.5(1.5,2) \mathrm{TeV}$, respectively (see [8] for the $m_{L}=0$ case).

Finally, in Fig.4 we describe the s-channel contribution to the $e^{-} e^{-} \rightarrow W^{-} W^{-}$process. We present the contribution of two doubly charged Higgs particles $\delta_{L}^{--}$and $\delta_{R}^{--}$which exist for example in the LR mode $\left.\right|^{2}$ Masses of the $\delta_{L, R}^{--}$ particles depend on $M_{W_{2}}$ [15] and for $M_{W_{2}}=1 \mathrm{TeV}$ we have (without fine tuning between parameters in the Higgs potential) $M_{\delta_{L}^{--}} \simeq 1600 \mathrm{GeV}$ and $M_{\delta_{R}^{--}} \simeq 3000 \mathrm{GeV}$. As $m_{\delta_{R}^{--}}>>m_{\delta_{L}^{--}}$the effect of $\delta_{R}^{--}$is negligible. In such circumstances our considerations are also valid for the SM enlarged by additional Higgs triplet and right handed neutrinos. Let us note that the contribution of the $\delta_{L}^{--}$resonance to the helicity amplitudes (Eq.(2)) is directly proportional to $m_{L}\left(m_{L}=\sum_{a} K_{a e}^{2} m_{a}\right.$, see e.g. [10]) and is invisible if only light neutrinos exist. If we take $m_{L}=0$ then the resonance disappears (solid line in Fig.4). If however $m_{L} \neq 0$ then its effect can be large. This is shown in

\footnotetext{
${ }^{2}$ Other aspects of doubly charged Higgs physics at an $e^{-} e^{-}$collider can be found in 14.
} 
Fig.4 where we take doubly charged Higgs' widths to be $\Gamma_{\delta_{L, R}^{--}}=\Gamma_{M_{W}} M_{\delta_{L, R}^{--}} / M_{W}$. Lines on this Figure present the cross sections for the case when all heavy neutrinos have the same CP eigenvalues. As it has already been discussed, $\sigma_{\max }$ depends strongly on $\omega^{2}$ in this case. We take $\omega^{2}=5 \cdot 10^{-5} \mathrm{TeV}^{-1}$, so $\mathrm{t}$ and $\mathrm{u}$ channels contributions to the cross section are very small (see Fig.1). This means that the large cross sections in Fig.4, even for energies far away from resonance region, are due to the $\delta_{L}^{--}$resonance. For example for $m_{L}=5 \mathrm{GeV}$ and $\sqrt{s}=1 \mathrm{TeV}$ the $\sigma_{\max } \simeq 40$ $\mathrm{fb}$ and the effect is caused almost exclusively by $\delta_{L}^{--}(1600)$ Higgs resonance. As the contribution of $\delta_{R}^{--}$to the cross section is not proportional to $m_{L}$ its effect can be large even for $m_{L}=0$ especially if its mass is around the C.M. energy. This case has been considered in [16].

This is shown explicitly in Fig.5 where s-channel contribution to the $e^{-} e^{-} \rightarrow W^{-} W^{-}$process as function of energy is presented. To extract the effect of the $\delta_{L}^{--}$resonance we compare the cross section $\sigma_{\max }$ for $\mathrm{t}$ and $\mathrm{u}$ channels only (short-dashed line) with the total cross section where t,u and s channels are added altogether (long-dashed lines) for $m_{L}=1 \mathrm{GeV}$ and $\eta_{C P}\left(N_{1}\right)=\eta_{C P}\left(N_{2}\right)$. We can see the huge influence of $\delta_{L}^{--}$resonance on the total cross section. Even for very high mass of $\delta_{L}^{--}\left(M_{\delta_{L}^{--}}=2000 \mathrm{GeV}, \sqrt{s}=1 \mathrm{TeV}\right) \sigma_{\max }$ is above the "discovery limit".

Solid lines in Fig.5 describe another case with $\eta_{C P}\left(N_{1}\right)=-\eta_{C P}\left(N_{2}\right)$. The upper one corresponds to the full cross section (s,t,u channels), the lower one is for a cross section without the s channel. As we can see for $m_{L}=-1 \mathrm{GeV}$, contributions of the $\mathrm{s}$ and $\mathrm{t}+\mathrm{u}$ channels are now comparable. The influence of $\delta_{L}^{--}$on the cross section depends on the $\delta_{L}^{--}$mass and width, and the value of the $m_{L}$ parameter. For the same mass and the same width of $\delta_{L}^{--}$its contribution to $\sigma_{\max }\left(e^{-} e^{-} \rightarrow W^{-} W^{-}\right)$can be very small, comparable or much bigger than the $\mathrm{t}+\mathrm{u}$ channels' part depending on the value of $m_{L}$.

\section{CONCLUSIONS}

We have analyzed the predictions for maximum possible value of the $e^{-} e^{-} \rightarrow W^{-} W^{-}$cross section in models with nonzero $m_{L}$. If we have only one heavy neutrino or more but with the same CP parities then the value of $\omega^{2}$ is crucial for the maximum of the cross section and $m_{L}$ does not have any visible influence. For the smallest value of $\omega^{2}\left(\leq 5 \times 10^{-5} \mathrm{TeV}^{-1}\right)$ predicted by some existing estimations (e.g. [13]) the cross section $\sigma_{\max }$ is too small to be measured in the future $e^{-} e^{-}$linear colliders unless $\delta_{L, R}^{--}$exist in the model.

However, for all other cases, the $m_{L} \neq 0$ changes substantially the $e^{-} e^{-} \rightarrow W^{-} W^{-}$cross section. Negative $m_{L}$ values move the limits on experimentally allowed neutrino mixings and masses. If there are two heavy neutrinos with opposite CP parities (or any number of them but with the lightest one having opposite CP parity with respect to all other ones) the value of $\sigma_{\max }$ can be substantial, much above the background level (e.g. for $\mathrm{M}=150 \mathrm{GeV}, \mathrm{A}=5$, $\sigma_{\max } \simeq 7 \mathrm{fb}$ ).

In another configuration of CP parities of heavy neutrinos $\left(\eta_{C P}\left(N_{1}\right)=\eta_{C P}\left(N_{2}\right)=-\eta_{C P}\left(N_{3}\right)\right)$ the largest $\sigma\left(e^{-} e^{-} \rightarrow\right.$ $\left.W^{-} W^{-}\right)$is obtained for $m_{L}=0$.

The most dramatic influence of the non-zero $m_{L}$ on $e^{-} e^{-} \rightarrow W^{-} W^{-}$is connected with the $\delta_{L}^{--}$resonance. For $m_{L}=0$ the contribution of this resonance to the process disappears. The $m_{L} \neq 0$ values cause the $\delta_{L}^{--}$to give large contribution even far away from on-peak energies. The contribution of the $\delta_{R}^{--}$to the cross section does not depend on the $m_{L}$ value.

\section{ACKNOWLEDGMENTS}

This work was supported by Polish Committee for Scientific Research under Grant No. PB659/P03/95/08 and University of Silesia internal Grant.

J.G. also appreciate the fellowship of the Foundation for Polish Science.

[1] Physics and technology of the Next Linear Collider: a report submitted to Snowmass '96 by NLC ZDR Design Group and NLC Physics Working Group (S. Kuhlman et al.), SLAC-R-0485, hep-ex/9605011.

[2] V. Barger, J.F. Beacom, K. Cheung, T. Han Phys. Rev. D50 (1994) 6704 and D52 (1995) 3815; J.F. Gunion and A. TofighiNiaki, Phys. Rev. D36 (1987) 2671 and D38 (1988) 1433; F. Cuypers, K. Kołodziej, O. Korakianitis and R. Rükl Phys. Lett. B325 (1994) 243. 
[3] J. Gluza and M. Zrałek, Phys. Lett. B362(1995)148.

[4] T. Rizzo, Phys.Lett.B116 (1982)23.

[5] C.A. Heusch and P. Minkowski, Nucl. Phys. B416 (1994) 3.

[6] G. Belanger, F. Boudjema, D. London and H. Nadeau, Phys. Rev. D53 (1996) 6292.

[7] D. London, G. Belanger and J.N. Ng, Phys.Lett. B188 (1987)155; J. Maalampi, A. Pietila and J. Vuori, Nucl.Phys. B381 (1992)544; C.A. Heusch and P. Minkowski, Phys.Lett.B374(1996)116; P. Helde, K. Huitu, J. Maalampi, M. Raidal, Nucl.Phys. B437 (1995)305; T. Rizzo Int. J. Mod. Phys. A11(1996)1563 and 1613; C.A. Heusch and P. Minkowski, SCIPP-96-41, hep-ph/9611353, B. Ananthanarayan and P. Minkowski, Phys. Lett. B373(1996)130.

[8] J. Gluza and M. Zrałek, Phys. Lett. B372 (1996) 259.

[9] J. Gluza, J. Maalampi, M. Raidal and M. Zrałek, Phys. Lett. B407 (1997) 45.

[10] J. Gluza and M. Zrałek, Phys. Rev. D52 (1995) 6238.

[11] See e.g., A. Djouadi, J. Ng and T.G. Rizzo, hep-ph/9504210; P. Langacker and D. London Phys. Rev. D38 (1988) 886 ; E. Nardi, E. Roulet, D. Tommasini Nucl. Phys. B386 (1992) 239; Phys. Lett. B344 (1995) 225; C.P. Burgess et al. Phys. Rev. D49 (1994) 6115.

[12] C. Greub and P. Minkowski, hep-ph/9612340.

[13] See e.g., J.D. Vergados, Phys. Rev. D28 (1983) 2887; Phys. Rep. 133 (1986) 1; A. Tomada, Rep. Prog. Phys. 54 (1991) 1; T. Bernatowicz et al., Phys. Rev. Lett. 69 (1992) 2341; A. Balysh et al. (Heidelberg-Moscow Coll.), Phys. Lett. B356 (1995) 450; M. Hirsch, H.V. Klapdor-Kleingrothaus and O. Panela, Phys. Lett. B374(1996)7.

[14] J.F. Gunion, Int. J. Mod. Phys. A11(1996)1551; J.F. Gunion, C. Loomis, K.T. Pitts, hep/ph/9610237.

[15] J. Gluza and M. Zrałek, Phys.Rev.D51(1995)4695.

[16] J. Gluza, Phys.Lett.B403(1997)304.

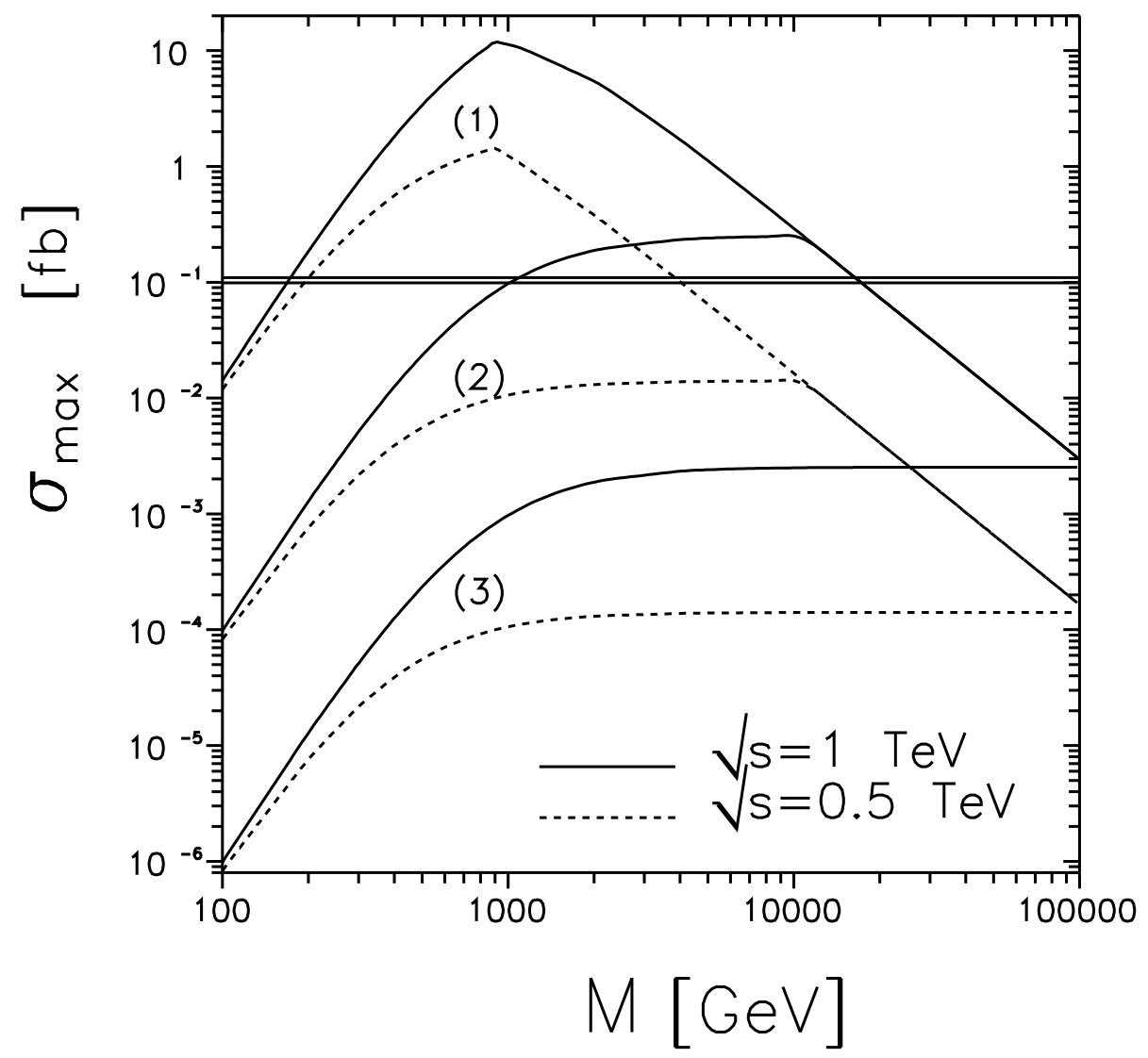

FIG. 1. The largest cross section for the $e^{-} e^{-} \rightarrow W^{-} W^{-}$process with any number of heavy neutrinos with the same CP parities. Dashed (solid) line is for $\sqrt{s}=0.5(1) \mathrm{TeV}$ energy, (1),(2),(3) stand for different $\omega^{2}$ values: $\omega^{2}=6 \cdot 10^{-3} \mathrm{TeV}-1(1)$; $\omega^{2}=5 \cdot 10^{-4} \mathrm{TeV}^{-1}(2) ; \omega^{2}=5 \cdot 10^{-5} \mathrm{TeV}^{-1}(3)$. Doubly solid line in this and next figures denotes a background level of this process. 


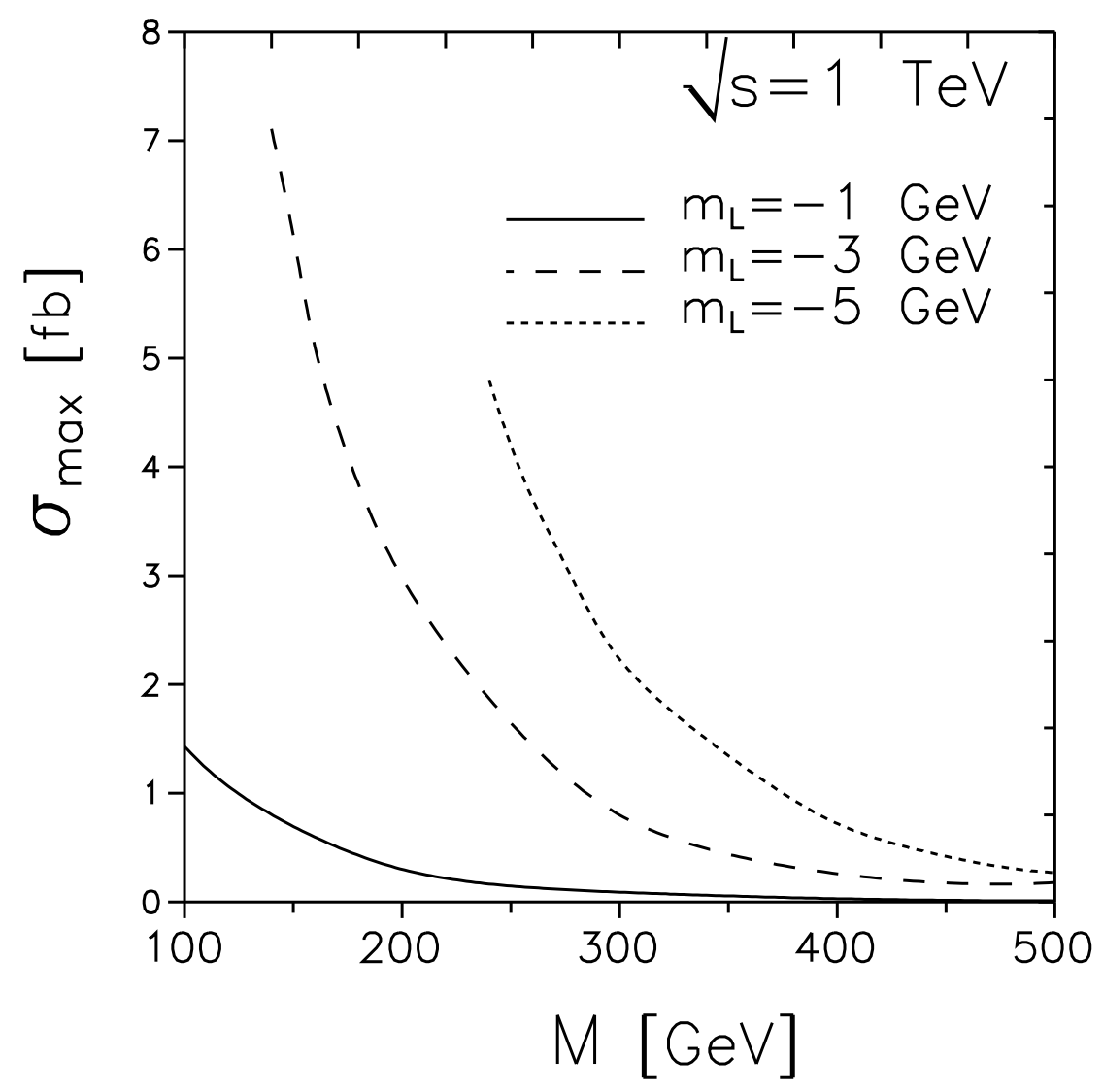

FIG. 2. Influence of the $m_{L}$ on $\sigma_{\max }$ for two heavy neutrinos with opposite $\mathrm{CP}$ parities for $\sqrt{s}=1 \mathrm{TeV}$ and $\mathrm{A}=5$ as function of M. Only negative $m_{L}$ values give substantial results in this case. 


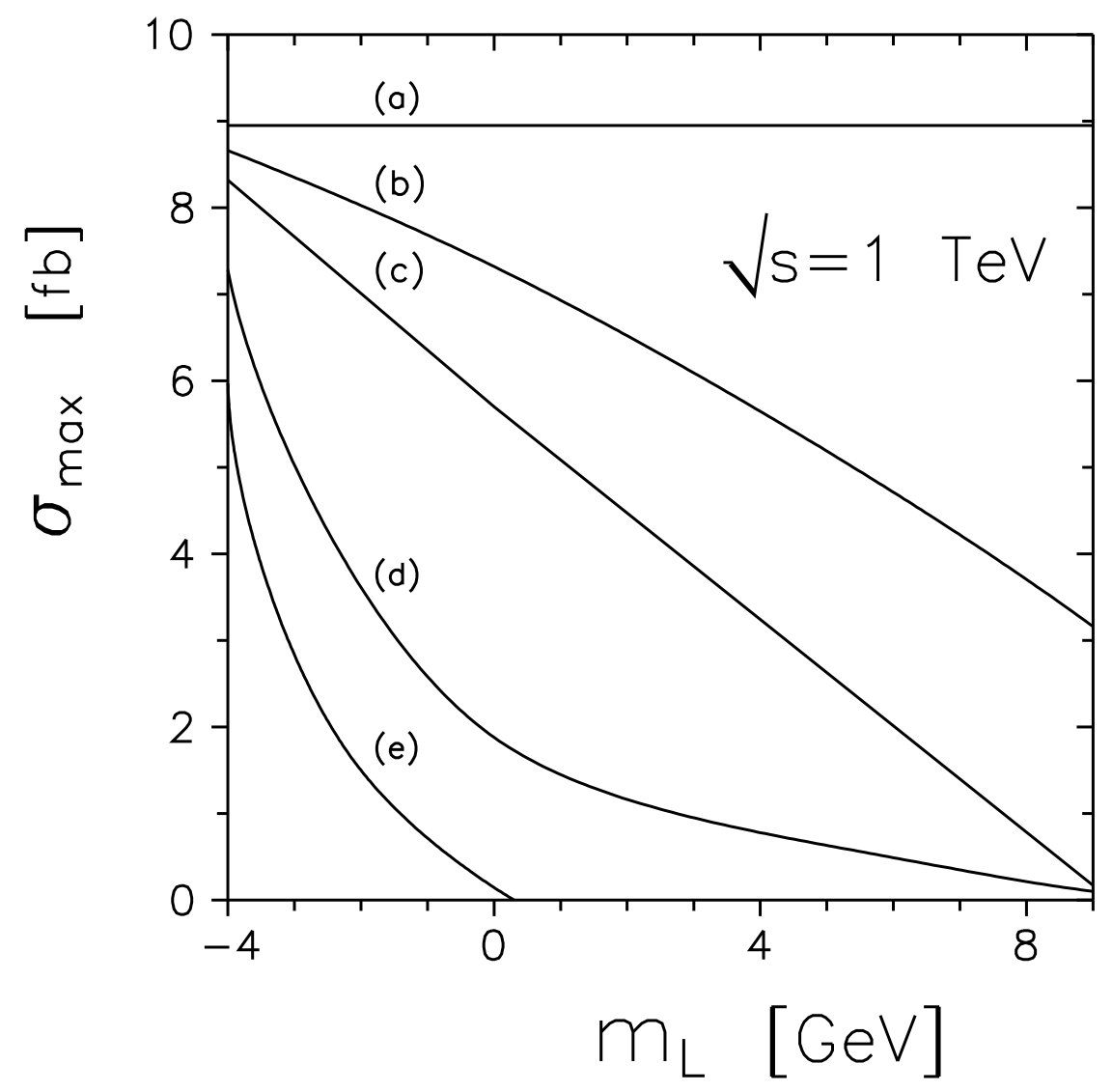

FIG. 3. The case of three heavy neutrinos with $\eta_{C P}\left(N_{1}\right)=\eta_{C P}\left(N_{2}\right)=-\eta_{C P}\left(N_{3}\right)$. The cross section as function of $m_{L}$ for different $\mathrm{A}, \mathrm{B}=10$ and $\mathrm{M}=100 \mathrm{GeV}$ is given. (a),(b),(c),(d),(e) are for $A=10^{6}, 100,50,20,10$, respectively. 


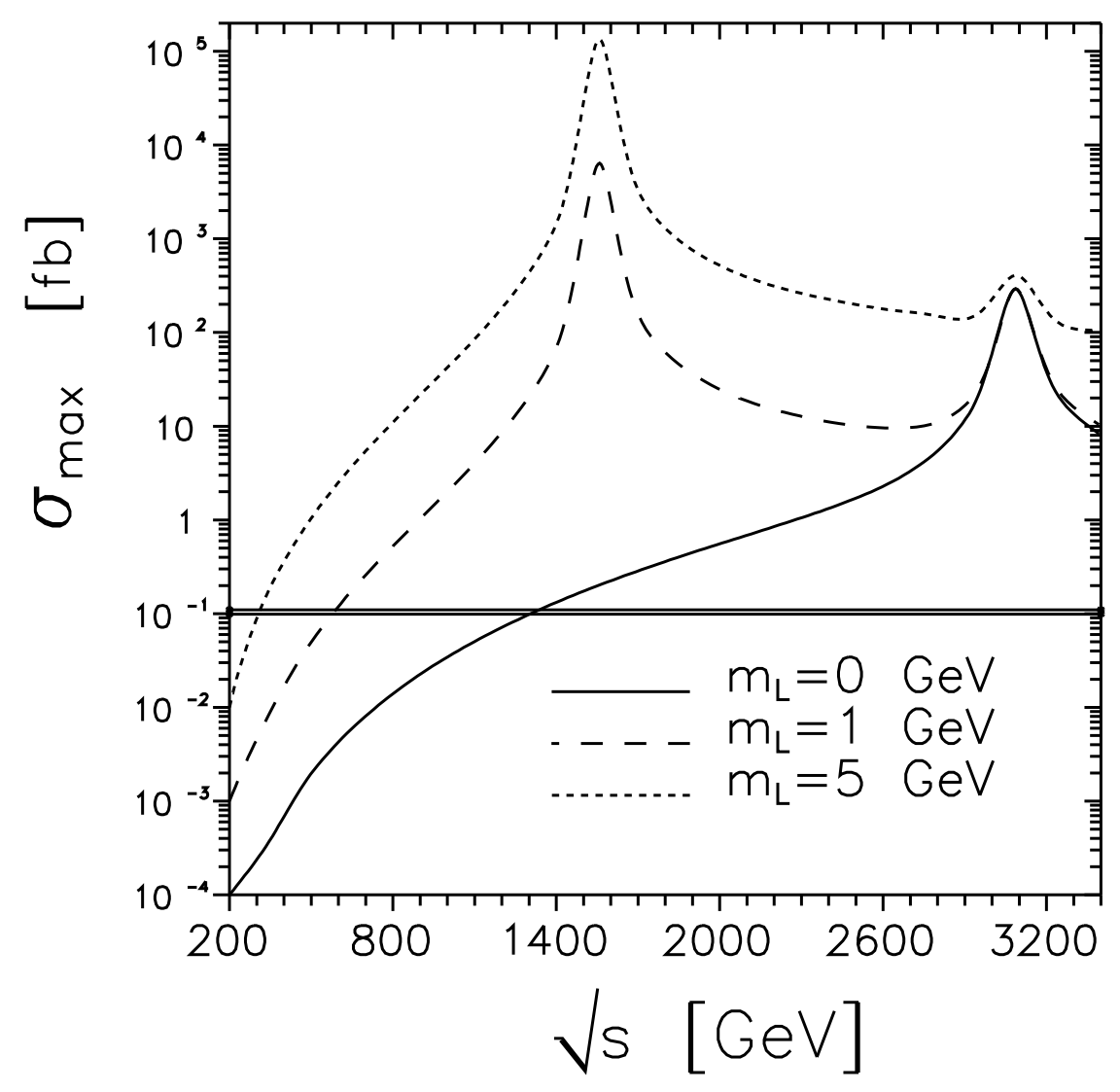

FIG. 4. Influence of the $m_{L}$ parameter on the s-channel $\delta_{L}^{--}$resonance. Solid, dashed and dotted lines are for $m_{L}=0,1,5$ $\mathrm{GeV}$, respectively. The $\mathrm{t}$ and $\mathrm{u}$ channel contributions are calculated for the same $\eta_{C P}$ eigenvalues of heavy neutrinos and $\omega^{2}=5 \cdot 10^{-5} \mathrm{TeV}^{-1}$. 


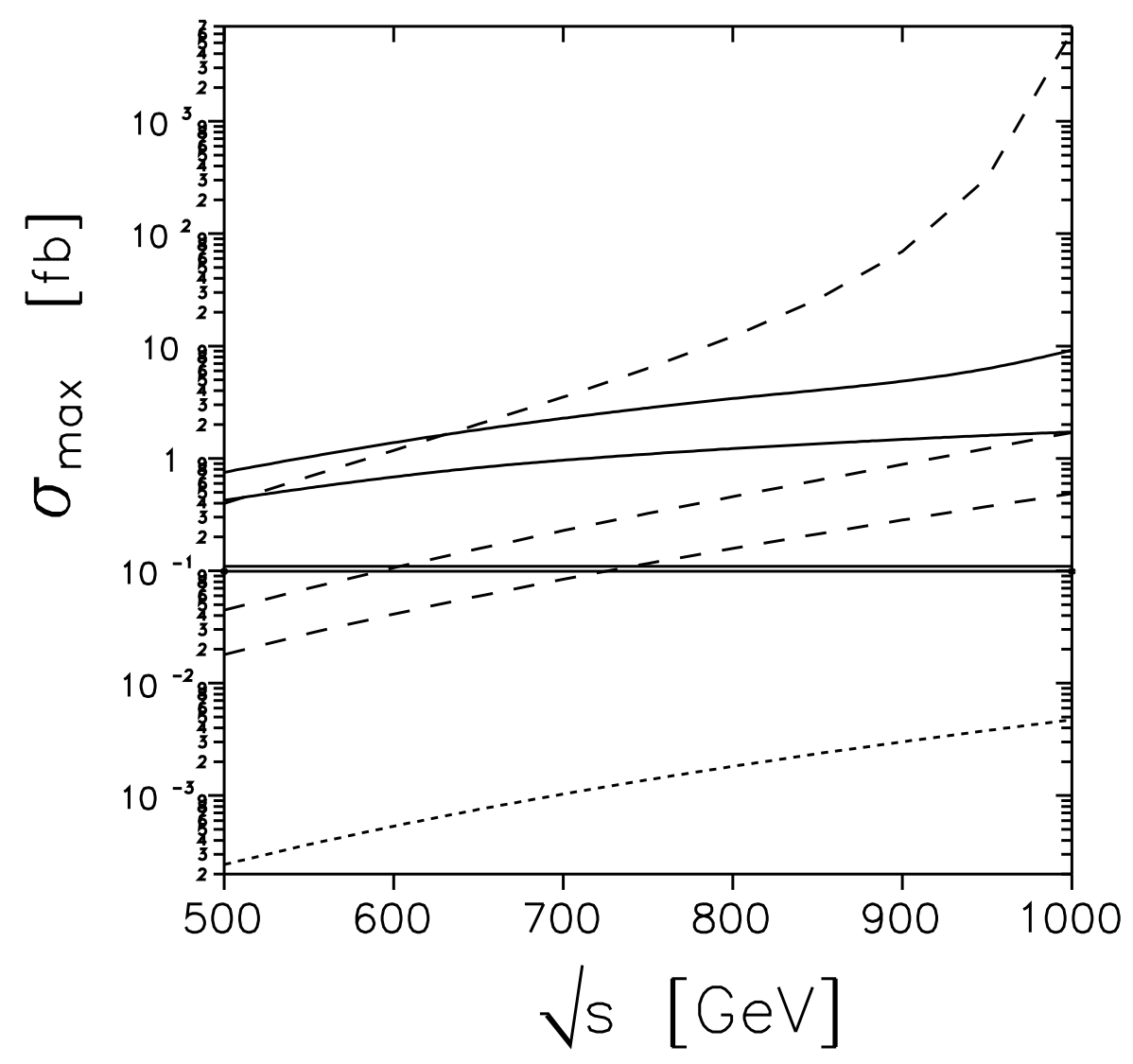

FIG. 5. Contribution of the $\delta_{L}^{--}$resonance to the $e^{-} e^{-} \rightarrow W^{-} W^{-}$process in the range of energies of the future linear lepton collider. Dashed lines are for $m_{L}=1 \mathrm{GeV}$ and the same CP parities of heavy neutrinos (case A), solid lines are for $m_{L}=-1$ $\mathrm{GeV}$ and $\eta_{C P}\left(N_{1}\right)=-\eta_{C P}\left(N_{2}\right)$ (case B). To show the s channel effect we present $\sigma_{\max }$ for $\mathrm{t}$ and $\mathrm{u}$ channels only (short dashed line for the A case and lower solid line for the B case) and for the full cross section with s,t and u channels altogether (long dashed lines for the A case and upper solid line for the B case). Long dashed lines are for $M_{\delta_{L}^{--}}=1000,1600,2000 \mathrm{GeV}$, respectively. The upper solid line is for $M_{\delta_{L}^{--}}=1600 \mathrm{GeV}$. 